\title{
STRENGTH OF A METAL SEVEN-LAYER RECTANGULAR PLATE WITH TRAPEZOIDAL CORRUGATED CORES
}

\author{
Ewa Magnucka-Blandzi, Zbigniew Walczak, Leszek Wittenbeck \\ Institute of Mathematics, Poznan University of Technology, Poznań, Poland \\ e-mail: ewa.magnucka-blandzi@put.poznan.pl,zbigniew.walczak@put.poznan.pl,leszek.wittenbeck@put.poznan.pl \\ MARCIN RODAK \\ Institute of Applied Mechanics, Poznan University of Technology, Poznan, Poland \\ e-mail: marcin.rodak@put.poznan.pl
}

\begin{abstract}
The subject of analytical and numerical investigations in this paper is a metal seven-layer rectangular plate with a trapezoidal corrugated main core and two trapezoidal corrugated cores of faces. The hypothesis of deformation of the normal to the middle surface of the plate after bending and field of displacements is formulated. The plate is simply supported on all its edges and subjected to a uniform pressure. Equations of equilibrium are derived based on the theorem of minimum total potential energy and are solved with the use of the Galerkin method. The influence of the trapezoidal corrugation pitch of the cores on the deflection and the equivalent stress is analysed.
\end{abstract}

Keywords: rectangular layered plate, corrugated cores, hypothesis of deformation, deflection, stresses

\section{Introduction}

The basic theoretical models of sandwich structures were formulated in the mid of the 20th century. Two decades later, Plantema (1966) and Allen (1969) elaborated first monographs devoted to bending, buckling and design problems of sandwich beams, plates and shells. Noor et al. (1996), Vinson (2001), Carrera (2003), Carrera and Brischetto (2009) presented a review of the problems related to computational models, applications and analysis of sandwich structures. Kazemahvazi and Zenkert (2009) developed an analytical model for the compressive and shear response of monolithic and hierarchical corrugated composite cores. Ji et al. (2010) described design procedures and the construction process of a glass fiber reinforced polymer corrugated-core sandwich bridge superstructure. Seong et al. (2010) introduced bi-directionally corrugated cores in order to reduce anisotropic behaviour of sandwich plates with open channel cores under the bending load. Magnucka-Blandzi (2011) described and solved analytically the problem of a simply supported rectangular sandwich plate under compression in plane. Poirier et al. (2013) proposed a methodology for designing lightweight laser-welded steel sandwich panels with superior structural performance. Jha et al. (2013) presented static analysis of orthotropic functionally graded elastic, rectangular and simply supported plates under transverse loads. Zhang et al. (2013) investigated compressive strengths and the dynamic response of corrugated sandwich plates with unfilled and foam filled sinusoidal plate cores. Magnucka-Blandzi and Magnucki (2014) determined analytically transverse shear moduli of corrugated cores in four different shapes. The influence of the corrugation shape on the shear modulus was studied. Lewinski et al. (2015) studied transverse shear moduli of two thin-walled trapezoidal corrugated cores of seven-layer sandwich plates. Magnucka-Blandzi et al. (2015) presented a mathematical modelling of the transverse shearing effect for sandwich beams with sinusoidal corrugated cores. 
The buckling and bending problems were solved. Magnucki et al. (2016) formulated two analytical models of a seven-layer steel beam with a transverse sinusoidal corrugated main core and two sandwich facings with steel foam cores, and solved the problem of bending and buckling. Cheon and Kim (2015) suggested an equivalent plate model to analyze the mechanical behaviour of corrugated-core sandwich panels under tensile and bending loads. Mantari and Granados (2015) presented a static analysis of functionally graded plates. In the paper, a simply supported square sandwich plate was subjected to a bi-sinusoidal load. Vaidya et al. (2015) investigated the response of sandwich steel beams with corrugated cores to quasi-static loading by employing experimental and computational approaches. A parametric study was also carried out on large-scale structural size beams of a few meters in length.

The subject of this study is a metal seven-layer rectangular plate with a trapezoidal corrugated main core and two trapezoidal corrugated cores of facings. The plate is simply supported and loaded with a uniformly distributed pressure.

\section{Mathematical modelling of a seven-layer plate}

\subsection{Displacements and strains}

A seven-layer rectangular plate with the trapezoidal corrugated main core, two inner flat sheets, two trapezoidal corrugated cores of the facings and two outer flat sheets is shown in Fig. 1. The plate is simply supported on all its edges and subjected to a uniform pressure $p_{0}$.

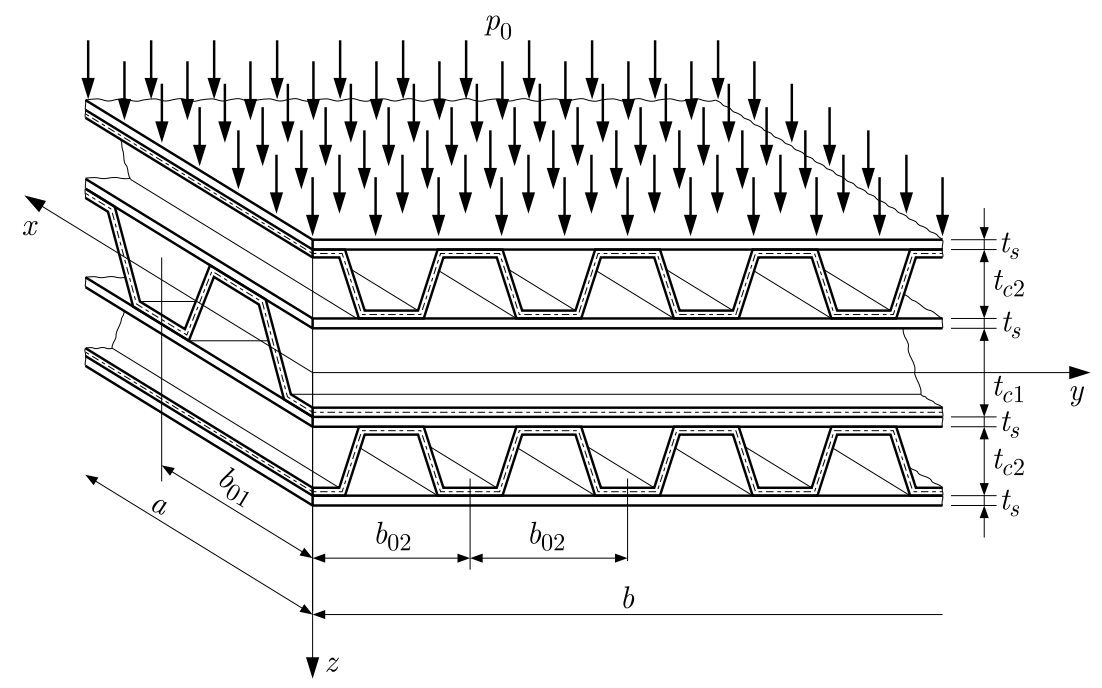

Fig. 1. Scheme of the seven-layer rectangular plate

The direction of the core facings corrugations is orthogonal to the one of the main core corrugation. Trapezoidal corrugations of the main core and facings cores are shown in Fig. 2.

(a)

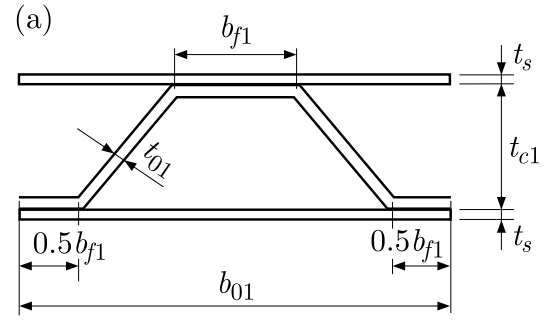

(b)

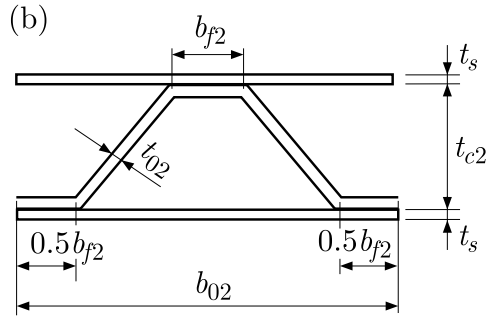

Fig. 2. Scheme of the corrugations of the (a) main core and (b) faces cores 
Taking into account the layered structures of the plate, it is easy to notice that the straight line normal to the middle plane of the plate before bending does not remain straight and normal after bending. The hypothesis is assumed that the straight line - normal after bending - takes a shape of a broken line (Fig. 3). The problem of the hypothesis for multi-layer structures was described, e.g. by Carrera (2003) and Magnucki et al. (2016).
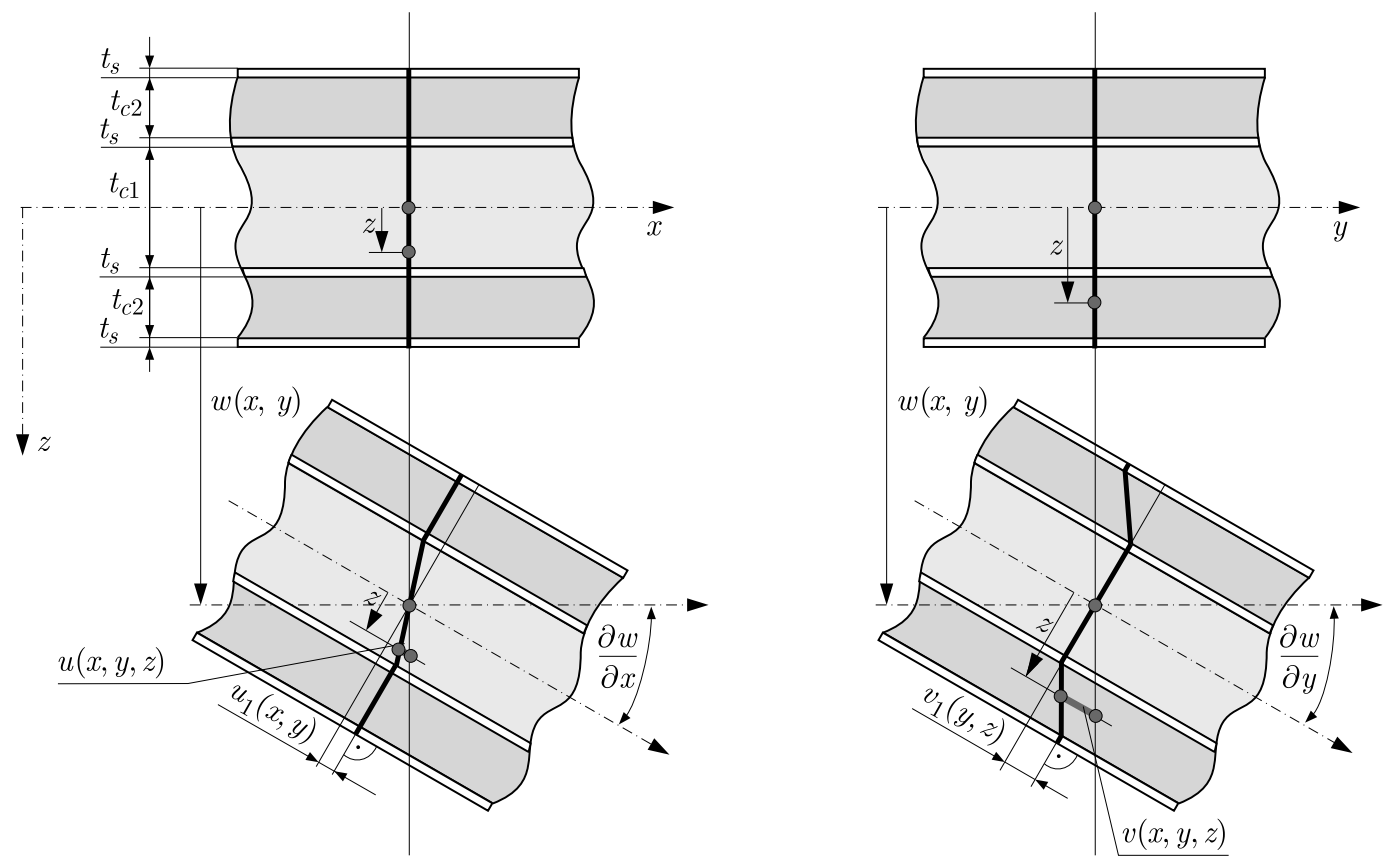

Fig. 3. Deformation of the normal to the middle plane of the plate

The displacements with consideration of the hypothesis are as follows:

1) outer flat sheets

- the upper sheet for $-\left(0.5+2 x_{1}+x_{2}\right) \leqslant \zeta \leqslant-\left(0.5+x_{1}+x_{2}\right)$

$$
u(x, y, z)=-t_{c 1}\left[\zeta \frac{\partial w}{\partial x}+\psi(x, y)\right] \quad v(x, y, z)=-t_{c 1}\left[\zeta \frac{\partial w}{\partial y}+x_{2} \phi(x, y)\right]
$$

- the lower sheet for $0.5+x_{1}+x_{2} \leqslant \zeta \leqslant 0.5+2 x_{1}+x_{2}$

$$
u(x, y, z)=-t_{c 1}\left[\zeta \frac{\partial w}{\partial x}-\psi(x, y)\right] \quad v(x, y, z)=-t_{c 1}\left[\zeta \frac{\partial w}{\partial y}-x_{2} \phi(x, y)\right]
$$

2) trapezoidal corrugated cores of the facings

- the upper core for $-\left(0.5+x_{1}+x_{2}\right) \leqslant \zeta \leqslant-\left(0.5+x_{1}\right)$

$$
\begin{aligned}
& u(x, y, z)=-t_{c 1}\left[\zeta \frac{\partial w}{\partial x}+\psi(x, y)\right] \\
& v(x, y, z)=-t_{c 1}\left\{\zeta \frac{\partial w}{\partial y}-\left[\zeta+\left(\frac{1}{2}+x_{1}\right)\right] \phi(x, y)\right\}
\end{aligned}
$$

- the lower core for $0.5+x_{1} \leqslant \zeta \leqslant 0.5+x_{1}+x_{2}$

$$
\begin{aligned}
& u(x, y, z)=-t_{c 1}\left[\zeta \frac{\partial w}{\partial x}-\psi(x, y)\right] \\
& v(x, y, z)=-t_{c 1}\left\{\zeta \frac{\partial w}{\partial y}-\left[\zeta-\left(\frac{1}{2}+x_{1}\right)\right] \phi(x, y)\right\}
\end{aligned}
$$


3) inner flat sheets

- the upper sheet for $-\left(0.5+x_{1}\right) \leqslant \zeta \leqslant-0.5$

$$
u(x, y, z)=-t_{c 1}\left[\zeta \frac{\partial w}{\partial x}+\psi(x, y)\right] \quad v(x, y, z)=-t_{c 1} \zeta \frac{\partial w}{\partial y}
$$

- the lower sheet for $0.5 \leqslant \zeta \leqslant 0.5+x_{1}$

$$
u(x, y, z)=-t_{c 1}\left[\zeta \frac{\partial w}{\partial x}-\psi(x, y)\right] \quad v(x, y, z)=-t_{c 1} \zeta \frac{\partial w}{\partial y}
$$

4) main corrugated core for $-0.5 \leqslant \zeta \leqslant 0.5$

$$
u(x, y, z)=-t_{c 1} \zeta\left[\frac{\partial w}{\partial x}-2 \psi(x, y)\right] \quad v(x, y, z)=-t_{c 1} \zeta \frac{\partial w}{\partial y}
$$

where $x_{1}=t_{s} / t_{c 1}, x_{2}=t_{c 2} / t_{c 1}$ are dimensionless parameters, $\xi=z / t_{c 1}$ - dimensionless coordinate, $t_{c 1}, t_{c 2}, t_{s}$ - thicknesses of the main core, facing cores and flat sheets (Fig. 2), $\psi(x, y)=u_{1}(x, y) / t_{c 1}, \phi(x, y)=v_{1}(x, y) / t_{c 2}$ - dimensionless functions of displacements, $u_{1}(x, y)$, $v_{1}(x, y)$ - displacements in the $x$ and $y$ directions, respectively, $w(x, y)$ - deflection (Fig. 3$)$ - deflections of each layer are equal and referenced to the middle plate layer, so $w(x, y, z) \equiv w(x, y)$ and $\varepsilon_{z} \equiv 0$.

Thus, the linear relations for strains are as follows:

1) outer flat sheets (upper/lower)

$$
\begin{aligned}
& \varepsilon_{x}^{(u / l)}=\frac{\partial u}{\partial x}=-t_{c 1}\left(\zeta \frac{\partial^{2} w}{\partial x^{2}} \pm \frac{\partial \psi}{\partial x}\right) \quad \varepsilon_{y}^{(u / l)}=\frac{\partial v}{\partial y}=-t_{c 1}\left(\zeta \frac{\partial^{2} w}{\partial y^{2}} \pm x_{2} \frac{\partial \phi}{\partial y}\right) \\
& \gamma_{x z}=\gamma_{y z}=0 \quad \gamma_{x y}^{(u / l)}=\frac{\partial u}{\partial y}+\frac{\partial v}{\partial x}=-t_{c 1}\left(2 \zeta \frac{\partial^{2} w}{\partial x \partial y} \pm \frac{\partial \psi}{\partial y} \pm x_{2} \frac{\partial \phi}{\partial x}\right)
\end{aligned}
$$

2) trapezoidal corrugated cores of the facings (upper/lower)

$$
\begin{aligned}
& \varepsilon_{x}^{(u / l)}=-t_{c 1}\left(\zeta \frac{\partial^{2} w}{\partial x^{2}} \pm \frac{\partial \psi}{\partial x}\right) \quad \varepsilon_{y}^{(u / l)}=-t_{c 1}\left\{\zeta \frac{\partial^{2} w}{\partial y^{2}}-\left[\zeta \pm\left(\frac{1}{2}+x_{1}\right)\right] \frac{\partial \phi}{\partial y}\right\} \\
& \gamma_{x z}=0 \quad \gamma_{y z}=\phi(x, y) \\
& \gamma_{x y}^{(u / l)}=-t_{c 1}\left\{2 \zeta \frac{\partial^{2} w}{\partial x \partial y} \pm \frac{\partial \psi}{\partial y}-\left[\zeta \pm\left(\frac{1}{2}+x_{1}\right)\right] \frac{\partial \phi}{\partial x}\right\}
\end{aligned}
$$

3) inner flat sheets (upper/lower)

$$
\begin{aligned}
& \varepsilon_{x}^{(u / l)}=-t_{c 1}\left(\zeta \frac{\partial^{2} w}{\partial x^{2}} \pm \frac{\partial \psi}{\partial x}\right) \quad \varepsilon_{y}^{(u / l)}=-t_{c 1} \zeta \frac{\partial^{2} w}{\partial y^{2}} \\
& \gamma_{x z}=\gamma_{y z}=0 \quad \gamma_{x y}^{(u / l)}=-t_{c 1}\left(2 \zeta \frac{\partial^{2} w}{\partial x \partial y} \pm \frac{\partial \psi}{\partial y}\right)
\end{aligned}
$$

The sign "+" refers to the upper facing $(u)$, and the sign "-" refers to the lower facing $(l)$. 4) main corrugated core

$$
\begin{aligned}
& \varepsilon_{x}=-t_{c 1} \zeta\left[\frac{\partial^{2} w}{\partial x^{2}}-2 \frac{\partial \psi}{\partial x}\right] \quad \varepsilon_{y}=-t_{c 1} \zeta \frac{\partial^{2} w}{\partial y^{2}} \\
& \gamma_{x z}=2 \psi(x, y) \quad \gamma_{y z}=0 \quad \gamma_{x y}=-2 t_{c 1} \zeta\left(\frac{\partial^{2} w}{\partial x \partial y}-\frac{\partial \psi}{\partial y}\right)
\end{aligned}
$$

Strains (2.8)-(2.11) make a basis for formulation of the elastic strain energy of the seven-layer plate. 


\subsection{Total potential energy of the plate}

The elastic strain energy of the plate is a sum of the energy of the individual layers

$$
U_{\varepsilon}^{(p l a t e)}=U_{\varepsilon}^{(s-o)}+U_{\varepsilon}^{(c-2)}+U_{\varepsilon}^{(s-i)}+U_{\varepsilon}^{(c-1)}
$$

Consecutive components of the sum are as follows:

1) energy of the outer flat sheets

$$
U_{\varepsilon}^{(s-o)}=\frac{t_{c 1}}{2} \int_{0}^{a} \int_{0}^{b}\left\{\int_{-\left(\frac{1}{2}+2 x_{1}+x_{2}\right)}^{-\left(\frac{1}{2}+x_{1}+x_{2}\right)}\left[\Phi_{\sigma, \varepsilon}^{(u, s-o)}\right] d \zeta+\int_{\frac{1}{2}+x_{1}+x_{2}}^{\frac{1}{2}+2 x_{1}+x_{2}}\left[\Phi_{\sigma, \varepsilon}^{(l, s-o)}\right] d \zeta\right\} d x d y
$$

where

$$
\Phi_{\sigma, \varepsilon}^{(u / l, s-o)}=\sigma_{x}^{(u / l)} \varepsilon_{x}^{(u / l)}+\sigma_{x}^{(u / l)} \varepsilon_{x}^{(u / l)}+\tau_{x y}^{(u / l)} \gamma_{x y}^{(u / l)}
$$

stresses (Hooke's law)

$$
\sigma_{x}^{(u / l)}=\frac{E}{1-\nu^{2}}\left(\varepsilon_{x}^{(u / l)}+\nu \varepsilon_{y}^{(u / l)}\right) \quad \tau_{x y}^{(u / l)}=\frac{E}{2(1+\nu)} \gamma_{x y}^{(u / l)}
$$

and strains - expressions (2.8).

Integration of expression (2.13) with respect to the coordinate $\zeta$ provides

$$
U_{\varepsilon}^{(s-o)}=\frac{E t_{c 1}^{3}}{1-\nu^{2}} \int_{0}^{a} \int_{0}^{b}\left(C_{2}^{(s-o)} f_{22}^{(s-o)}-c_{1}^{(s-o)} f_{12}^{(s-o)}+x_{1} f_{11}^{(s-o)}\right) d x d y
$$

where

$$
\begin{aligned}
& c_{2}^{(s-o)}=\frac{1}{12}\left[28 x_{1}^{2}+18 x_{1}\left(1+2 x_{2}\right)+3\left(1+2 x_{2}\right)^{2}\right] x_{1} \quad c_{1}^{(s-o)}=\left(1+3 x_{1}+2 x_{2}\right) x_{1} \\
& f_{22}^{(s-o)}=\left(\frac{\partial^{2} w}{\partial x^{2}}\right)^{2}+2 \nu \frac{\partial^{2} w}{\partial x^{2}} \frac{\partial^{2} w}{\partial y^{2}}+\left(\frac{\partial^{2} w}{\partial y^{2}}\right)^{2}+2(1-\nu)\left(\frac{\partial^{2} w}{\partial x \partial y}\right)^{2} \\
& f_{12}^{(s-o)}=\left(\frac{\partial^{2} w}{\partial x^{2}}+\nu \frac{\partial^{2} w}{\partial y^{2}}\right) \frac{\partial \psi}{\partial x}+x_{2}\left(\nu \frac{\partial^{2} w}{\partial x^{2}}+\frac{\partial^{2} w}{\partial y^{2}}\right) \frac{\partial \phi}{\partial y}+(1-\nu)\left(\frac{\partial \psi}{\partial y}+x_{2} \frac{\partial \phi}{\partial x}\right) \frac{\partial^{2} w}{\partial x \partial y} \\
& f_{11}^{(s-o)}=\left(\frac{\partial \psi}{\partial x}\right)^{2}+\frac{1-\nu}{2}\left(\frac{\partial \psi}{\partial y}\right)^{2}+x_{2}\left[2 \nu \frac{\partial \psi}{\partial x} \frac{\partial \phi}{\partial y}+(1-\nu) \frac{\partial \phi}{\partial x} \frac{\partial \psi}{\partial y}\right] \\
&+x_{2}^{2}\left[\frac{1-\nu}{2}\left(\frac{\partial \phi}{\partial x}\right)^{2}+\left(\frac{\partial \phi}{\partial y}\right)^{2}\right]
\end{aligned}
$$

2) energy of the corrugated cores of the facings

$$
U_{\varepsilon}^{(c-2)}=\frac{1}{2} \int_{0}^{a} \int_{0}^{b}\left\{\frac{1}{b_{02}} \int_{A_{T r}}\left[\Phi_{\sigma, \varepsilon}^{(u, c-2)}\right] d A_{T r}^{(c-2)}+\frac{1}{b_{02}} \int_{A_{T r}}\left[\Phi_{\sigma, \varepsilon}^{(l, c-2)}\right] d A_{T r}^{(c-2)}\right\} d x d y
$$

where

$$
\Phi_{\sigma, \varepsilon}^{(u / l, c-2)}=\sigma_{x}^{(u / l)} \varepsilon_{x}^{(u / l)}+\sigma_{y}^{(u / l)} \varepsilon_{y}^{(u / l)}+\tau_{x y}^{(u / l)} \gamma_{x y}^{(u / l)}+\tau_{y z}^{(u / l)} \gamma_{y z}^{(u / l)}
$$

stresses

$$
\begin{array}{ll}
\sigma_{x}^{(u / l)} & =E \varepsilon_{x}^{(u / l)} \\
\tau_{y z}^{(u / l)} & =G_{y z}^{(c-2)} \gamma_{y z}^{(u / l)}
\end{array} \quad \sigma_{y}^{(u / l)}=E_{y}^{(c-2)} \varepsilon_{y}^{(u / l)} \quad \tau_{x y}^{(u / l)}=G_{x y}^{(c-2)} \gamma_{x y}^{(u / l)}
$$

and strains - expressions (2.9). 
The area of one pitch of the trapezoidal corrugated cross section (Fig. 2)

$$
A_{T r}^{(c-2)}=2 t_{c 2}^{2} x_{02}\left(x_{f 2} x_{b 2}+\widetilde{s}_{a 2}\right)
$$

where $x_{02}=t_{02} / t_{c 2}, x_{f 2}=b_{f 2} / b_{02}, x_{b 2}=b_{02} / t_{c 2}$ are dimensionless parameters, $\widetilde{s}_{a 2}$ - dimensionless length of one pitch - trapezoid

$$
\tilde{s}_{a 2}=\sqrt{\left(1-x_{02}\right)^{2}+x_{b 2}^{2}\left(\frac{1}{2}-x_{f 2}\right)^{2}}
$$

Integration of expression (2.17) provides

$$
U_{\varepsilon}^{(c-2)}=E t_{c 1}^{3} \int_{0}^{a} \int_{0}^{b}\left[f_{22}^{(c-2)}+\widetilde{E}_{y}^{(c-2)} f_{12}^{(c-2)}+\widetilde{G}_{x y}^{(c-2)} f_{11}^{(c-2)}+\widetilde{G}_{y z}^{(c-2)} f_{10}^{(c-2)}\right] d x d y
$$

where

$$
\begin{aligned}
& f_{22}^{(c-2)}=c_{2 x}^{(c-2)}\left(\frac{\partial^{2} w}{\partial x^{2}}\right)^{2}-2 c_{1 x}^{(c-2)} \frac{\partial^{2} w}{\partial x^{2}} \frac{\partial \psi}{\partial x}+c_{0 x}^{(c-2)}\left(\frac{\partial \psi}{\partial x}\right)^{2} \\
& \widetilde{E}_{y}^{(c-2)}=\frac{x_{b 2} x_{02}^{3}}{2\left(3 x_{f 2} x_{b 2}+\widetilde{s}_{a 2}\right)\left(1-x_{02}\right)^{2}} \quad f_{10}^{(c-2)}=x_{2} \frac{\phi^{2}(x, y)}{t_{c 1}^{2}} \\
& f_{12}^{(c-2)}=C_{2 y}^{(c-2)}\left(\frac{\partial^{2} w}{\partial y^{2}}\right)^{2}-C_{1 y}^{(c-2)} \frac{\partial^{2} w}{\partial y^{2}} \frac{\partial \phi}{\partial y}+C_{0 y}^{(c-2)}\left(\frac{\partial \phi}{\partial y}\right)^{2} \\
& f_{11}^{(c-2)}=4 C_{2 y}^{(c-2)}\left(\frac{\partial^{2} w}{\partial x \partial y}\right)^{2}+C_{0 y}^{(c-2)}\left(\frac{\partial \phi}{\partial x}\right)^{2}-\left(2 C_{1 y}^{(c-2)} \frac{\partial \phi}{\partial x}+c_{1 x y}^{(c-2)} \frac{\partial \psi}{\partial y}\right) \frac{\partial^{2} w}{\partial x \partial y} \\
&+x_{2}\left(x_{2} \frac{\partial \phi}{\partial x}+\frac{\partial \psi}{\partial y}\right) \frac{\partial \psi}{\partial y} \quad C_{1 x}^{(c-2)}=x_{2} x_{02}\left(1+2 x_{1}+x_{2}\right)\left(x_{f 2}+\frac{\widetilde{s}_{a 2}}{x_{b 2}}\right) \\
& C_{2 x}^{(c-2)}=\frac{1}{2} x_{2} x_{02}\left[x_{2}^{2}\left(1-x_{02}\right)^{2}\left(x_{f 2}+\frac{\widetilde{s}_{a 2}}{3 x_{b 2}}\right)+\left(1+2 x_{1}+x_{2}\right)\left(x_{f 2}+\frac{\widetilde{s}_{a 2}}{x_{b 2}}\right)\right] \\
& G_{x y}^{(c-2)}=\frac{x_{02}}{2(1+\nu)}=\frac{1}{2} x_{2}^{2}\left(1+2 x_{1}+\frac{4}{3} x_{2}\right) \\
& C_{0 x}^{(c-2)}=2 x_{2} x_{02}\left(x_{f 2}+\frac{\widetilde{s}_{a 2}}{x_{b 2}}\right) \quad C_{2 y}^{(c-2)}=x_{2}\left[x_{1}^{2}+x_{1}\left(1+x_{2}\right)+\frac{1}{4}\left(1+2 x_{2}+\frac{4}{3} x_{2}^{2}\right)\right] \\
& C_{0 y}^{(c-2)}=\frac{1}{3} x_{2}^{3} \quad \widetilde{G}_{y z}^{(c-2)}=\frac{2}{\left(1-\nu^{2}\right) x_{b 2} f_{v}}\left(\frac{x_{02}}{\widetilde{s}_{a 2}}\right)^{3} \\
& C_{1 x y}^{(c-2)}=2 x_{2}\left(1+2 x_{1}+x_{2}\right) \quad
\end{aligned}
$$

details in Lewinski et al. (2015)

3) energy of the inner flat sheets

$$
U_{\varepsilon}^{(s-i)}=\frac{t_{c 1}}{2} \int_{0}^{a} \int_{0}^{b}\left\{\int_{-\left(\frac{1}{2}+x_{1}\right)}^{-\frac{1}{2}}\left[\Phi_{\sigma, \varepsilon}^{(u, s-i)}\right] d \zeta+\int_{\frac{1}{2}}^{\frac{1}{2}+x_{1}}\left[\Phi_{\sigma, \varepsilon}^{(l, s-i)}\right] d \zeta\right\} d x d y
$$

where

$$
\Phi_{\sigma, \varepsilon}^{(u / l, s-i)}=\sigma_{x}^{(u / l)} \varepsilon_{x}^{(u / l)}+\sigma_{x}^{(u / l)} \varepsilon_{x}^{(u / l)}+\tau_{x y}^{(u / l)} \gamma_{x y}^{(u / l)}
$$

stresses (Hooke's law)

$$
\sigma_{x}^{(u / l)}=\frac{E}{1-\nu^{2}}\left(\varepsilon_{x}^{(u / l)}+\nu \varepsilon_{y}^{(u / l)}\right) \quad \tau_{x y}^{(u / l)}=\frac{E}{2(1+\nu)} \gamma_{x y}^{(u / l)}
$$

and strains - expressions (2.10). 
Integration of expression (2.22) with respect to the coordinate $\zeta$ provides

$$
U_{\varepsilon}^{(s-i)}=\frac{E t_{c 1}^{3}}{1-\nu^{2}} \int_{0}^{a} \int_{0}^{b}\left(C_{2}^{(s-i)} f_{22}^{(s-i)}-C_{1}^{(s-i)} f_{12}^{(s-i)}+x_{1} f_{11}^{(s-i)}\right) d x d y
$$

where

$$
\begin{array}{lcc}
C_{2}^{(s-i)}=\frac{1}{4}\left(1+2 x_{1}+\frac{4}{3} x_{1}^{2}\right) x_{1} & C_{1}^{(s-i)}=\left(1+x_{1}\right) x_{1} \quad f_{22}^{(s-i)}=f_{22}^{(s-o)} \\
f_{12}^{(s-i)}=\left(\frac{\partial^{2} w}{\partial x^{2}}+\nu \frac{\partial^{2} w}{\partial y^{2}}\right) \frac{\partial \psi}{\partial x}+(1-\nu) \frac{\partial^{2} w}{\partial x \partial y} \frac{\partial \psi}{\partial y} & f_{11}^{(s-i)}=\left(\frac{\partial \psi}{\partial x}\right)^{2}+\frac{1-\nu}{2}\left(\frac{\partial \psi}{\partial y}\right)^{2}
\end{array}
$$

4) energy of the main corrugated core

$$
U_{\varepsilon}^{(c-1)}=\frac{1}{2 b_{01}} \int_{0}^{a} \int_{0}^{b}\left\{\int_{A_{T r}}\left[\Phi_{\sigma, \varepsilon}^{(c-1)}\right] d A_{T r}^{(c-1)}\right\} d x d y
$$

where

$$
\Phi_{\sigma, \varepsilon}^{(c-1)}=\sigma_{x} \varepsilon_{x}+\sigma_{y} \varepsilon_{y}+\tau_{x y} \gamma_{x y}+\tau_{x z} \gamma_{x z}
$$

stresses

$$
\sigma_{x}=E_{x}^{(c-1)} \varepsilon_{x} \quad \sigma_{y}=E \varepsilon_{y} \quad \tau_{x y}=G_{x y}^{(c-1)} \gamma_{x y} \quad \tau_{x z}=G_{x z}^{(c-1)} \gamma_{x z}
$$

and strains - expressions (2.11).

The area of one pitch of the trapezoidal corrugated cross section (Fig. 2)

$$
A_{T r}^{(c-1)}=2 t_{c 1}^{2} x_{01}\left(x_{f 1} x_{b 1}+\widetilde{s}_{a 1}\right)
$$

where $x_{01}=t_{01} / t_{c 1}, x_{f 1}=b_{f 1} / b_{01}, x_{b 1}=b_{01} / t_{c 1}$ are dimensionless parameters, $\widetilde{s}_{a 1}$ - dimensionless length of one pitch - trapezoid

$$
\widetilde{s}_{a 1}=\sqrt{\left(1-x_{01}\right)^{2}+x_{b 1}^{2}\left(\frac{1}{2}-x_{f 1}\right)^{2}}
$$

Integration of expression (2.30) provides

$$
U_{\varepsilon}^{(c-1)}=E t_{c 1}^{3} \int_{0}^{a} \int_{0}^{b}\left(\frac{1}{24} \widetilde{E}_{x}^{(c-1)} f_{22}^{(c-1)}+\frac{1}{24} \widetilde{E}_{y}^{(c-1)} f_{12}^{(c-1)}+\frac{1}{6} \widetilde{G}_{x y}^{(c-1)} f_{11}^{(c-1)}+2 G_{x z}^{(c-1)} f_{10}^{(c-1)}\right) d x d y
$$

where

$$
\begin{aligned}
f_{22}^{(c-1)} & =\left(\frac{\partial^{2} w}{\partial x^{2}}\right)^{2}-4 \frac{\partial^{2} w}{\partial x^{2}} \frac{\partial \psi}{\partial x}+4\left(\frac{\partial \psi}{\partial x}\right)^{2} \quad f_{12}^{(c-1)}=\left(\frac{\partial^{2} w}{\partial y^{2}}\right)^{2} \\
f_{10}^{(c-1)} & =\frac{\psi^{2}(x, y)}{t_{c 1}^{2}} \quad f_{11}^{(c-1)}=\left(\frac{\partial^{2} w}{\partial x \partial y}\right)^{2}-2 \frac{\partial^{2} w}{\partial x \partial y} \frac{\partial \psi}{\partial y}+\left(\frac{\partial \psi}{\partial y}\right)^{2} \\
\widetilde{E}_{x}^{(c-1)} & =\frac{x_{b 1} x_{01}^{3}}{2\left(x_{f 1} x_{b 1}+\widetilde{s}_{a 1}\right)} \quad \quad \widetilde{G}_{x y}^{(c-1)}=\frac{x_{01}}{2(1+\nu)} \\
\widetilde{E}_{y}^{(c-1)} & =2 \frac{x_{01}}{x_{b 1}}\left(1-x_{01}\right)^{2}\left(3 x_{f 1} x_{b 1}+\widetilde{s}_{a 1}\right) \quad \widetilde{G}_{x z}^{(c-1)}=\frac{1-x_{01}}{4\left(1-\nu^{2}\right) x_{b 1} f_{u}}\left(\frac{x_{01}}{\widetilde{s}_{a 1}}\right)^{3}
\end{aligned}
$$

detail in Lewinski et al. (2015). 
The work of the load, a uniformly distributed pressure $p_{0}$, is in the following form

$$
W=\int_{0}^{a} \int_{0}^{b} p_{0} w(x, y) d x d y
$$

The total potential energy is a sum of elastic strain energy (2.12) and work (2.31).

\section{Equations of equilibrium and its solution}

The principle of minimum total potential energy

$$
\delta\left(U_{\varepsilon}^{(\text {plate })}-W\right)=0
$$

where $U_{\varepsilon}^{(\text {plate) }}$ is the elastic strain energy of the plate (2.12) and $W$ is the work of the load (2.35).

The system of the equations of equilibrium - three partial differential equations derived based on principle (3.1) is in the following form

$$
\Re_{w}^{(s-o-i)}+\Re_{w}^{(c-2)}+\Re_{w}^{(c-1)}=\frac{p_{0}}{E t_{c 1}^{3}}
$$

where

$$
\begin{aligned}
& \Re_{w}^{(s-o-i)}=\frac{1}{1-\nu^{2}}\left\{2\left(C_{2}^{(s-o)}+C_{2}^{(s-i)}\right) \nabla^{4} w-C_{1}^{(s-o)}\left[\frac{\partial}{\partial x}\left(\nabla^{2} \psi\right)+x_{2} \frac{\partial}{\partial y}\left(\nabla^{2} \phi\right)\right]\right. \\
& \left.-C_{1}^{(s-i)} \frac{\partial}{\partial x}\left(\nabla^{2} \psi\right)\right\} \\
& \Re_{w}^{(c-2)}=2 \Re_{w, w}^{(c-2)}-\Re_{w, \psi}^{(c-2)}-\Re_{w, \phi}^{(c-2)} \\
& \Re_{w, w}^{(c-2)}=C_{2 x}^{(c-2)} \frac{\partial^{4} w}{\partial x^{4}}+C_{2 y}^{(c-2)}\left(4 \widetilde{G}_{x y}^{(c-2)} \frac{\partial^{4} w}{\partial x^{2} \partial y^{2}}+\widetilde{E}_{y}^{(c-2)} \frac{\partial^{4} w}{\partial y^{4}}\right) \\
& \Re_{w, \psi}^{(c-2)}=\frac{\partial}{\partial x}\left(2 C_{1 x}^{(c-2)} \frac{\partial^{2} \psi}{\partial x^{2}}+C_{1 x y}^{(c-2)} \widetilde{G}_{x y}^{(c-2)} \frac{\partial^{2} \psi}{\partial y^{2}}\right) \\
& \Re_{w, \phi}^{(c-2)}=C_{1 y}^{(c-2)} \frac{\partial}{\partial x}\left(2 \widetilde{G}_{x y}^{(c-2)} \frac{\partial^{2} \phi}{\partial x^{2}}+\widetilde{E}_{y}^{(c-2)} \frac{\partial^{2} \phi}{\partial y^{2}}\right) \\
& \Re_{w}^{(c-1)}=\frac{1}{12} \Re_{w, w}^{(c-1)}-\frac{1}{6} \Re_{w, \psi}^{(c-1)} \quad \Re_{w, w}^{(c-1)}=\widetilde{E}_{x}^{(c-1)} \frac{\partial^{4} w}{\partial x^{4}}+4 \widetilde{G}_{x y}^{(c-1)} \frac{\partial^{4} w}{\partial x^{2} \partial y^{2}}+\widetilde{E}_{y}^{(c-1)} \frac{\partial^{4} w}{\partial y^{4}} \\
& \Re_{w, \psi}^{(c-1)}=\frac{\partial}{\partial x}\left(\widetilde{E}_{x}^{(c-1)} \frac{\partial^{2} \psi}{\partial x^{2}}+2 \widetilde{G}_{x y}^{(c-1)} \frac{\partial^{2} \psi}{\partial y^{2}}\right) \quad \nabla^{4} w=\frac{\partial^{4} w}{\partial x^{4}}+2 \frac{\partial^{4} w}{\partial x^{2} \partial y^{2}}+\frac{\partial^{4} w}{\partial y^{4}}
\end{aligned}
$$

and

$$
\Re_{\psi}^{(s-o-i)}+\Re_{\psi}^{(c-2)}+\Re_{\psi}^{(c-1)}=0
$$

where

$$
\begin{aligned}
& \Re_{\psi}^{(s-o-i)}=\frac{1}{1-\nu^{2}}\left\{\left(C_{1}^{(s-o)}+C_{1}^{(s-i)}\right) \frac{\partial}{\partial x}\left(\nabla^{2} w\right)-2 x_{1}\left[2 \frac{\partial^{2} \psi}{\partial x^{2}}+(1-\nu) \frac{\partial^{2} \psi}{\partial y^{2}}\right]\right. \\
& \left.\quad-x_{1} x_{2}(1+\nu) \frac{\partial^{2} \phi}{\partial x \partial y}\right\} \\
& \Re_{\psi}^{(c-2)}=\frac{\partial}{\partial x}\left(2 C_{1 x}^{(c-2)} \frac{\partial^{2} w}{\partial x^{2}}+C_{1 x y}^{(c-2)} \widetilde{G}_{x y}^{(c-2)} \frac{\partial^{2} w}{\partial y^{2}}\right)-2\left(C_{0 x}^{(c-2)} \frac{\partial^{2} \psi}{\partial x^{2}}+x_{2} \widetilde{G}_{x y}^{(c-2)} \frac{\partial^{2} \psi}{\partial y^{2}}\right) \\
& \quad-x_{2}^{2} \widetilde{G}_{x y}^{(c-2)} \frac{\partial^{2} \phi}{\partial x \partial y} \\
& \Re_{\psi}^{(c-1)}=\frac{1}{6} \frac{\partial}{\partial x}\left(\widetilde{E}_{x}^{(c-1)} \frac{\partial^{2} w}{\partial x^{2}}+2 \widetilde{G}_{x y}^{(c-1)} \frac{\partial^{2} w}{\partial y^{2}}\right)-\frac{1}{3}\left(\widetilde{E}_{x}^{(c-1)} \frac{\partial^{2} \psi}{\partial x^{2}}+\widetilde{G}_{x y}^{(c-1)} \frac{\partial^{2} \psi}{\partial y^{2}}\right)-4 \widetilde{G}_{x z}^{(c-1)} \frac{\psi(x, y)}{t_{c 1}^{2}}
\end{aligned}
$$


and

$$
\Re_{\phi}^{(s-o-i)}+\Re_{\phi}^{(c-2)}=0
$$

where

$$
\begin{aligned}
& \Re_{\phi}^{(s-o-i)}=\frac{1}{1-\nu^{2}}\left\{x_{2} C_{1}^{(s-o)} \frac{\partial}{\partial y}\left(\nabla^{2} w\right)-x_{1} x_{2}^{2}\left[(1-\nu) \frac{\partial^{2} \phi}{\partial x^{2}}+2 \frac{\partial^{2} \phi}{\partial y^{2}}\right]-x_{1} x_{2}(1+\nu) \frac{\partial^{2} \psi}{\partial x \partial y}\right\} \\
& \Re_{\phi}^{(c-2)}=\Re_{\phi, w}^{(c-2)}-x_{2}^{2} \widetilde{G}_{x y}^{(c-2)} \frac{\partial^{2} \psi}{\partial x \partial y}-\Re_{\phi, \phi}^{(c-2)} \\
& \Re_{\phi, w}^{(c-2)}=C_{1 y}^{(c-2)} \frac{\partial}{\partial y}\left(2 \widetilde{G}_{x y}^{(c-2)} \frac{\partial^{2} w}{\partial x^{2}}+\widetilde{E}_{y}^{(c-2)} \frac{\partial^{2} w}{\partial y^{2}}\right) \\
& \Re_{\phi, \phi}^{(c-2)}=2 C_{0 y}^{(c-2)}\left(\widetilde{G}_{x y}^{(c-2)} \frac{\partial^{2} \phi}{\partial x^{2}}+\widetilde{E}_{y}^{(c-2)} \frac{\partial^{2} \phi}{\partial y^{2}}\right)-2 x_{2} \widetilde{G}_{y z}^{(c-2)} \frac{\phi(x, y)}{t_{c 1}^{2}} \\
& \nabla^{2} w=\frac{\partial^{2} w}{\partial x^{2}}+\frac{\partial^{2} w}{\partial y^{2}}
\end{aligned}
$$

Three equations of equilibrium (3.2), (3.3) and (3.4) with three unknown functions $w(x, y)$, $\psi(x, y)$ and $\phi(x, y)$ are approximately solved assuming three unknown functions in the forms

$$
\begin{aligned}
& w(x, y)=w_{a} \sin \frac{\pi x}{a} \sin \frac{\pi y}{b} \quad \psi(x, y)=\psi_{a} \cos \frac{\pi x}{a} \sin \frac{\pi y}{b} \\
& \phi(x, y)=\phi_{a} \sin \frac{\pi x}{a} \cos \frac{\pi y}{b}
\end{aligned}
$$

where $w_{a}, \psi_{a}, \phi_{a}$ are parameters of the functions, $a, b$ - sizes of the plate (Fig. 1).

Substituting these functions into equations (3.2), (3.3) and (3.4) and using the Galerkin method, three algebraic equations are obtained

$$
\begin{aligned}
& \alpha_{11} w_{a}-\alpha_{12} \frac{b}{\pi} \psi_{a}-\alpha_{13} \frac{a}{\pi} \phi_{a}=\frac{16}{\pi^{3}} \frac{a^{2} b^{2}}{t_{c 1}^{3}} \frac{p_{0}}{e} \quad \alpha_{21} \frac{\pi}{a} w_{a}-\alpha_{22} \psi_{a}-\alpha_{23} \phi_{a}=0 \\
& \alpha_{31} \frac{\pi}{b} w_{a}-\alpha_{32} \psi_{a}-\alpha_{33} \phi_{a}=0
\end{aligned}
$$

where the dimensionless elements

$$
\begin{aligned}
\alpha_{11} & =\alpha_{11}^{(1)}+\alpha_{11}^{(2)}+\alpha_{11}^{(3)} \quad \alpha_{11}^{(1)}=\frac{2}{1-\nu^{2}}\left(C_{2}^{(s-o)}+C_{2}^{(s-i)}\right)\left(\frac{b}{a}+\frac{a}{b}\right)^{2} \\
\alpha_{23} & =x_{2}\left(\frac{x_{1}}{1-\nu}+x_{2} \widetilde{G}_{x y}^{(c-2)}\right) \quad \alpha_{11}^{(3)}=\frac{1}{12}\left[\widetilde{E}_{x}^{(c-1)}\left(\frac{b}{a}\right)^{2}+4 \widetilde{G}_{x y}^{(c-1)}+\widetilde{E}_{y}^{(c-1)}\left(\frac{a}{b}\right)^{2}\right] \\
\alpha_{11}^{(2)} & =2\left[C_{2 x}^{(c-2)}\left(\frac{b}{a}\right)^{2}+C_{2 y}^{(c-2)}\left(4 \widetilde{G}_{x y}^{(c-2)}+\widetilde{E}_{y}^{(c-2)}\left(\frac{a}{b}\right)^{2}\right)\right] \\
\alpha_{12} & =\frac{1}{1-\nu^{2}}\left(C_{1}^{(s-o)}+C_{1}^{(s-i)}\right)\left(\frac{b}{a}+\frac{a}{b}\right)+2 C_{1 x}^{(c-2)} \frac{b}{a}+C_{1 x y}^{(c-2)} \widetilde{G}_{x y}^{(c-2)} \frac{a}{b} \\
& +\frac{1}{6}\left(\widetilde{E}_{x}^{(c-1)} \frac{b}{a}+2 \widetilde{G}_{x y}^{(c-1)} \frac{a}{b}\right) \\
\alpha_{13} & =\frac{x_{2}}{1-\nu^{2}} C_{1}^{(s-o)}\left(\frac{b}{a}+\frac{a}{b}\right)+C_{1 y}^{(c-2)}\left(2 \widetilde{G}_{x y}^{(c-2)} \frac{b}{a}+\widetilde{E}_{y}^{(c-2)} \frac{a}{b}\right) \\
\alpha_{21} & =\alpha_{12} \quad \alpha_{32}=\alpha_{23} \\
\alpha_{22} & =\frac{2 x_{1}}{1-\nu^{2}}\left[2 \frac{b}{a}+(1-\nu) \frac{a}{b}\right]+2\left(C_{0 x}^{(c-2)} \frac{b}{a}+x_{2} \widetilde{G}_{x y}^{(c-2)} \frac{a}{b}\right)+\frac{1}{3}\left(\widetilde{E}_{x}^{(c-1)} \frac{b}{a}+\widetilde{G}_{x y}^{(c-1)} \frac{a}{b}\right) \\
& +\frac{4}{\pi^{2}} \widetilde{G}_{x z}^{(c-1)} \frac{a b}{t_{c 1}^{2}} \\
\alpha_{33} & =\frac{x_{1} x_{2}^{2}}{1-\nu^{2}}\left[(1-\nu) \frac{b}{a}+2 \frac{a}{b}\right]+2 C_{0 y}^{(c-2)}\left(\widetilde{G}_{x y}^{(c-2)} \frac{b}{a}+\widetilde{E}_{y}^{(c-2)} \frac{a}{b}\right)+\frac{2 x_{2}}{\pi^{2}} \widetilde{G}_{y z}^{(c-2)} \frac{a b}{t_{c 1}^{2}}
\end{aligned}
$$


Solving equations (3.6) one obtains

$$
w_{a}=\frac{16}{\pi^{6} \alpha_{w}} \frac{a^{2} b^{2}}{t_{c 1}^{3}} \frac{p_{0}}{E} \quad \psi_{a}=\frac{16}{\pi^{5}} \frac{\alpha_{\psi}}{\alpha_{w}} \frac{a^{2} b}{t_{c 1}^{3}} \frac{p_{0}}{E} \quad \phi_{a}=\frac{16}{\pi^{5}} \frac{\alpha_{\phi}}{\alpha_{w}} \frac{a^{2} b}{t_{c 1}^{3}} \frac{p_{0}}{E}
$$

where

$$
\begin{array}{ll}
\alpha_{w} & =\alpha_{11}-\left(\alpha_{\psi} \alpha_{12}+\alpha_{\phi} \alpha_{13}\right) \quad \alpha_{\psi}=\frac{b \alpha_{21} \alpha_{33}-a \alpha_{31} \alpha_{23}}{a\left(\alpha_{22} \alpha_{33}-\alpha_{23}^{2}\right)} \\
\alpha_{\phi} & =\frac{a \alpha_{31} \alpha_{22}-b \alpha_{21} \alpha_{32}}{b\left(\alpha_{22} \alpha_{33}-\alpha_{23}^{2}\right)}
\end{array}
$$

The stresses on the outer sheets and in the middle of the plate, for $\zeta_{o}=\mp\left(0.5+2 x_{1}+x_{2}\right)$ and $x=a / 2, y=b / 2$ are

$$
\begin{aligned}
\sigma_{x} & =\frac{1}{1-\nu^{2}}\left[\left(\frac{b}{a}+\nu \frac{a}{b}\right) \zeta_{o} \pm\left(\alpha_{\psi}+\nu x_{2} \alpha_{\phi}\right)\right] \frac{16}{\pi^{4} \alpha_{w}} \frac{a b}{t_{c 1}^{2}} p_{0} \\
\sigma_{y} & =\frac{1}{1-\nu^{2}}\left[\left(\frac{a}{b}+\nu \frac{b}{a}\right) \zeta_{o} \pm\left(\nu \alpha_{\psi}+x_{2} \alpha_{\phi}\right)\right] \frac{16}{\pi^{4} \alpha_{w}} \frac{a b}{t_{c 1}^{2}} p_{0}
\end{aligned}
$$

and the equivalent stress (Huber-Mises-Hencky)

$$
\sigma_{e q}=\sqrt{f_{\sigma x}^{2}-f_{\sigma x} f_{\sigma y}+f_{\sigma y}^{2}} \frac{16}{\pi^{4}\left(1-\nu^{2}\right) \alpha_{w}} \frac{a b}{t_{c 1}^{2}} p_{0}
$$

where

$$
f_{\sigma x}=\left(\frac{b}{a}+\nu \frac{a}{b}\right) \zeta_{o} \pm\left(\alpha_{\psi}+\nu x_{2} \alpha_{\phi}\right) \quad f_{\sigma y}=\left(\frac{a}{b}+\nu \frac{b}{a}\right) \zeta_{o} \pm\left(\nu \alpha_{\psi}+x_{2} \alpha_{\phi}\right)
$$

\section{Finite element model of the seven-layer plate}

A family of simply supported rectangular plates of dimensions $2024 \mathrm{~mm} \times 2000 \mathrm{~mm}$ subjected to a uniform load of $0.01 \mathrm{MPa}$ has been considered. The linear static analysis was carried out using the finite element software ABAQUS. A quarter of the rectangular plate was modeled. The linear S4R shell elements were placed at the mid-surface of the plate layers (Fig. 4).

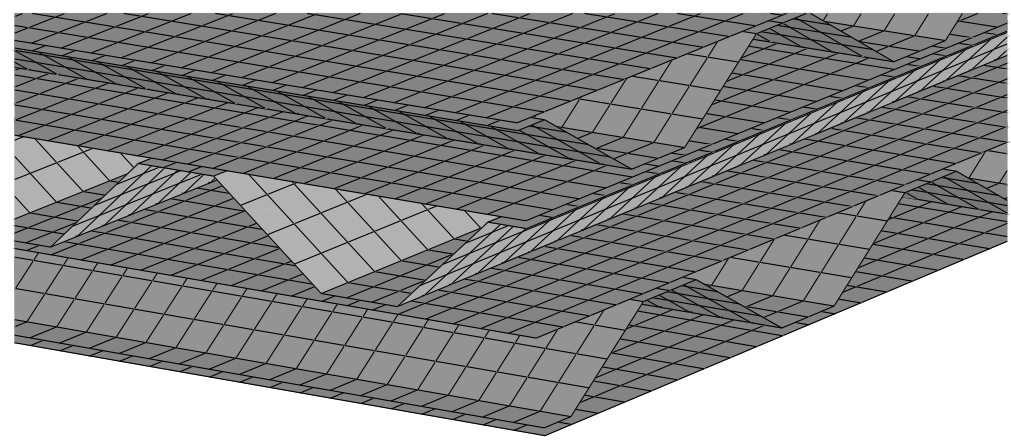

Fig. 4. The meshing scheme of a simply supported plate

The mesh density study was carried out to refine the global mesh size to $4 \mathrm{~mm}$. The mesh convergence plot for the maximum deflection in the middle of the top face sheet is presented in Fig. 5. 


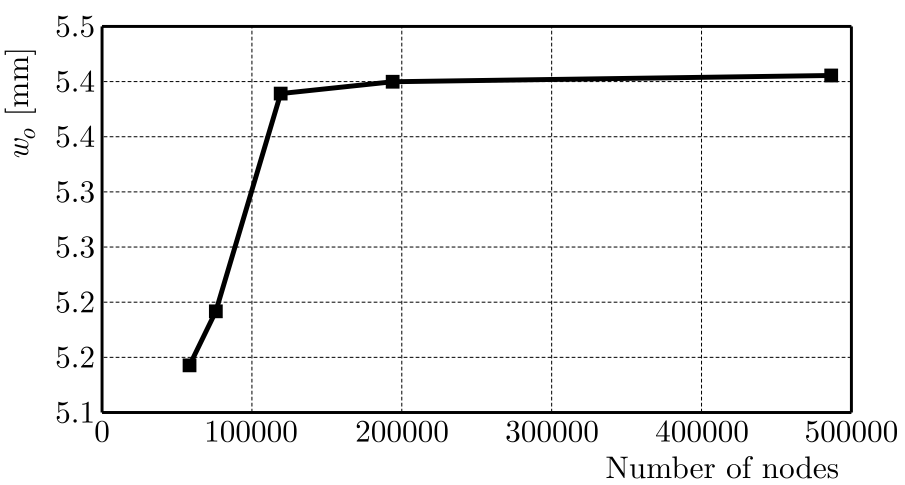

Fig. 5. The mesh convergence plot

Perfect bonding between the cores and the flat sheets was assumed. The interaction between flanges of the cores and the flat sheets was provided with the use of the tie constraint. The flanges of the cores were slave surfaces and the flat sheets were master surfaces.

The boundary conditions were imposed only to edges of the flat sheets (master surfaces) each edge was simply supported. The implementation of the symmetry and the simply supported boundary conditions on a quarter of the plate is schematically shown in Fig. 6 .

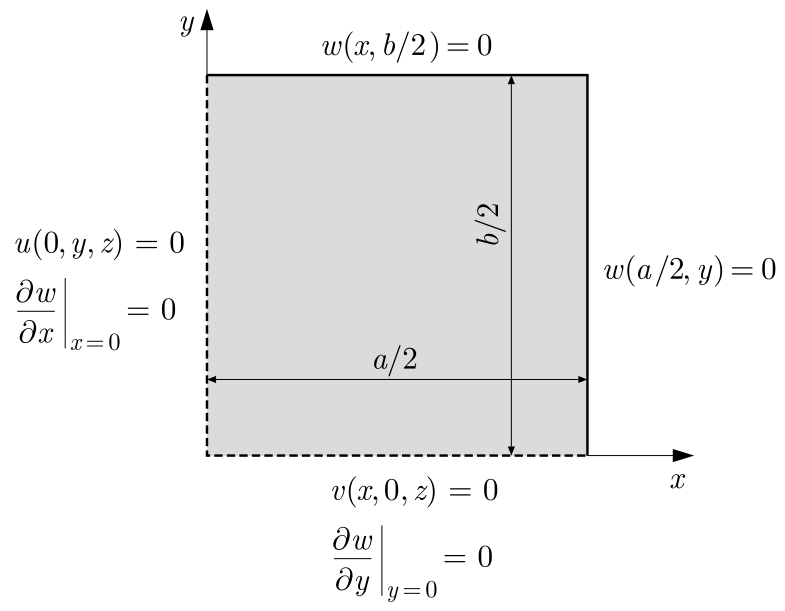

Fig. 6. The scheme of boundary conditions

\section{Results of numerical calculations of deflection and stresses of the plate}

The aim of these calculations was to verify the results obtained through the linear finite element analysis with those obtained through an analytical method. The maximum deflection and the equivalent stress of the family of seven-layer rectangular plates, using both analytical and finite element methods, was evaluated. The results of the parametric studies for changes of $b_{02}$ and $b_{01}$ are collected in Case 1 and Case 2, respectively.

Case 1. The study for constant area of the trapezoidal corrugation of the facing core $A_{\text {Total }}^{(c-2)}=n A_{T r}^{(c-2)}$, where $n$ is the number of the corrugations and $A_{T r}^{(c-2)}(2.20)$ is the area of one pitch of the trapezoidal corrugated cross section. The numerical calculations are carried out for the rectangular plate with the following sizes: $a=2024 \mathrm{~mm}$, $b=2000 \mathrm{~mm}, t_{s}=0.8 \mathrm{~mm}, t_{c 1}=11.2 \mathrm{~mm}, t_{01}=0.8 \mathrm{~mm}, b_{01}=46 \mathrm{~mm}, b_{f 1}=10 \mathrm{~mm}$, $t_{c 2}=9.2 \mathrm{~mm}, b_{f 2}=8 \mathrm{~mm}, A_{\text {Total }}^{(c-2)}=1811.83 \mathrm{~mm}^{2}, p_{0}=0.01 \mathrm{MPa}$, and material constants 
$E=2 \cdot 10^{5} \mathrm{MPa}, \nu=0.3$. The results of the calculations are presented in Table 1 . The values in the ABAQUS columns in Table 1 enclosed in parentheses are percentage differences with respect to the analytical ones (the absolute value of the relative deviation).

Table 1. The deflection and the equivalent stresses of the plate for the first case $A_{\text {Total }}^{(c-2)}=$ const

\begin{tabular}{|c|c|c|c|c|c|c|}
\hline \multirow{2}{*}{$n$} & \multirow{2}{*}{$\begin{array}{c}b_{02} \\
{[\mathrm{~mm}]}\end{array}$} & \multirow{2}{*}{$\begin{array}{c}t_{02} \\
{[\mathrm{~mm}]}\end{array}$} & $\begin{array}{c}\text { Analytical } \\
{[\mathrm{mm}]}\end{array}$ & $\begin{array}{c}\text { ABAQUS } \\
{[\mathrm{mm}]}\end{array}$ & $\begin{array}{c}\text { Analytical } \\
{[\mathrm{MPa}]}\end{array}$ & $\begin{array}{c}\text { ABAQUS } \\
{[\mathrm{MPa}]}\end{array}$ \\
\hline \hline 50 & 40.0 & 0.8 & 5.30 & $5.41(2.1 \%)$ & 59.4 & $60.01(1.0 \%)$ \\
\hline 60 & 33.333 & 0.751 & 5.33 & $5.43(1.9 \%)$ & 59.7 & $60.86(1.9 \%)$ \\
\hline 70 & 28.571 & 0.6968 & 5.38 & $5.48(1.8 \%)$ & 60.1 & $62.40(3.8 \%)$ \\
\hline 80 & 25.0 & 0.6409 & 5.43 & $5.53(1.7 \%)$ & 60.5 & $63.44(4.7 \%)$ \\
\hline 90 & 22.222 & 0.5867 & 5.51 & $5.61(1.8 \%)$ & 60.9 & $64.94(6.4 \%)$ \\
\hline 100 & 20.0 & 0.5363 & 5.61 & $5.72(1.9 \%)$ & 61.2 & $66.29(8.0 \%)$ \\
\hline
\end{tabular}

Case 2. The study for constant area of the trapezoidal corrugation of the main core $A_{\text {Total }}^{(c-1)}=m A_{T r}^{(c-1)}$, where $m$ is the number of the corrugations and $A_{T r}^{(c-1)}(2.29)$ is the area of one pitch of the trapezoidal corrugated cross section. The numerical calculations are carried out for the rectangular plate with the following sizes: $a=2024 \mathrm{~mm}, b=2000 \mathrm{~mm}$, $t_{s}=0.8 \mathrm{~mm}, t_{c 1}=11.2 \mathrm{~mm}, b_{f 1}=10 \mathrm{~mm}, t_{c 2}=9.2 \mathrm{~mm}, t_{02}=0.8 \mathrm{~mm}, b_{02}=40 \mathrm{~mm}$, $b_{f 2}=8 \mathrm{~mm}, A_{\text {Total }}^{(c-1)}=1876.03 \mathrm{~mm}^{2}, p_{0}=0.01 \mathrm{MPa}, E=2 \cdot 10^{5} \mathrm{MPa}, \nu=0.3$. The results of the calculations are presented in Table 2. The values in the ABAQUS columns in Table 2 enclosed in parentheses are percentage differences with respect to the analytical ones (the absolute value of the relative deviation).

Table 2. The deflection and the equivalent stresses of the plate for the second case $A_{\text {Total }}^{(c-1)}=$ const

\begin{tabular}{|c|c|c|c|c|c|c|}
\hline \multirow{2}{*}{$n$} & \multirow{2}{*}{$\begin{array}{c}b_{01} \\
{[\mathrm{~mm}]}\end{array}$} & \multirow{2}{*}{$\begin{array}{c}t_{01} \\
{[\mathrm{~mm}]}\end{array}$} & $\begin{array}{c}\text { Analytical } \\
{[\mathrm{mm}]}\end{array}$ & $\begin{array}{c}\text { ABAQUS } \\
{[\mathrm{mm}]}\end{array}$ & $\begin{array}{c}\text { Analytical } \\
{[\mathrm{MPa}]}\end{array}$ & $\begin{array}{c}\text { ABAQUS } \\
{[\mathrm{MPa}]}\end{array}$ \\
\hline \hline 44 & 46.0 & 0.8 & 5.30 & $5.41(2.1 \%)$ & 59.34 & $60.01(1.1 \%)$ \\
\hline 54 & 37.481 & 0.7349 & 5.33 & $5.43(1.8 \%)$ & 59.44 & $60.07(1.1 \%)$ \\
\hline 64 & 31.625 & 0.6652 & 5.41 & $5.49(1.5 \%)$ & 59.73 & $60.47(1.2 \%)$ \\
\hline 74 & 27.351 & 0.5973 & 5.65 & $5.65(0.0 \%)$ & 60.55 & $61.33(1.3 \%)$ \\
\hline 84 & 24.095 & 0.5245 & 6.48 & $5.98(8.0 \%)$ & 63.90 & $63.00(1.4 \%)$ \\
\hline
\end{tabular}

\section{Conclusions}

As a conclusion, it can be said that the results obtained through the analytical and the numerical method are consistent with each other. It proves that the broken-line hypotheses assumed for deformation of the cross-section in $x$ and $y$ directions are sufficient for evaluating the plate deflection and equivalent stresses. It can also be seen that decreasing of the parameter $b_{01}$ has much more influence on the increase of the maximum deflection of the plate. This effect is due to a significant change in the shear rigidity of the plate.

Acknowledgements The project was funded by the National Science Centre allocated on the basis of the decision number DEC-2013/09/B/ST8/00170. 


\section{References}

1. Allen H.G., 1969, Analysis and Design of Structural Sandwich Panels, Pergamon Press, Oxford, London, Edinburgh, New York, Toronto, Sydney, Paris, Braunschweig

2. CARrera E., 2003, Historical review of Zig-Zag theories for multi-layered plates and shells, Applied Mechanics Reviews, 56, 3, 287-308

3. Carrera E., Brischetto S., 2009, A survey with numerical assessment of classical and refined theories for the analysis of sandwich plates, Applied Mechanics Reviews, 62, 1-010803, 1-17

4. Cheon Y.J., Kim H.G., 2015, An equivalent plate model for corrugated-core sandwich panels, Journal of Mechanical Science and Technology, 29, 3, 1217-1223

5. Jha D.K., Kant T., Singh R.K., 2013, Stress analysis of transversely loaded functionally graded plates with a higher order shear and normal deformation theory, ASCE Journal of Engineering Mechanics, 139, 12, 1663-1680

6. Ji H.S., Song W., MA Z.J., 2010, Design, test and field application of a GFRP corrugated-core sandwich bridge, Engineering Structures, 32, 2814-2824

7. Kazemahvazi S., Zenkert D., 2009, Corrugated all-composite sandwich structures. Part 1: Modeling, Composite Science and Technology, 69, 7, 913-919

8. Lewinski J., Magnucka-Blandzi E., SzyC W., 2015, Determination of shear modulus of elasticity for thin-walled trapezoidal corrugated cores of seven-layer sandwich plates, Engineering Transactions, 63, 4, 421-438

9. MagnuCKA-Blandzi E., 2011, Mathematical modelling of a rectangular sandwich plate with a metal foam core, Journal of Theoretical and Applied Mechanics, 49, 2, 439-455

10. Magnucka-Blandzi E., Magnucki K., 2014, Transverse shear modulus of elasticity for thinwalled corrugated cores of sandwich beams. Theoretical study, Journal of Theoretical and Applied Mechanics, 52, 4, 971-980

11. Magnucka-Blandzi E., Magnucki K., Wittenbeck L., 2015, Mathematical modelling of shearing effect for sandwich beams with sinusoidal corrugated cores, Applied Mathematical Modelling, 39, 9, 2796-2808

12. Magnucki K., Magnucka-Blandzi E., Wittenbeck L., 2016, Elastic bending and buckling of a steel composite beam with corrugated main core and sandwich faces - Theoretical study, Applied Mathematical Modelling, 40, 2, 1276-1286

13. Mantari J.L., Granados E.V., 2015, A refined FSDT for the static analysis of functionally graded sandwich plates, Thin-Walled Structures, 90, 150-158

14. Noor A.K., Burton W.S., Bert C.W., 1996, Computational models for sandwich panels and shells, Applied Mechanics Reviews, 49, 3, 155-199

15. Plantema F.J., 1966, Sandwich construction - the bending and buckling of sandwich beams, plates, and shells, John Wiley \& Sons, New York, London, Sydney

16. Poirier J.D., Vel S.S., Caccese V., 2013, Multi-objective optimization of laser-welded steel sandwich panels for static loads using a genetic algorithm, Engineering Structures, 49, 508-524

17. Seong D.Y., Jung C.G., Yang D.Y., Moon K.J., Ahn D.G., 2010, Quasi-isotropic bending responses of metallic sandwich plates with bi-directionally corrugated cores, Materials and Design, 31, 6, 2804-2812

18. Vaidya S., Zhang L., Maddala D., Hegert R., Wright J.T., Shukla A., Kim J.H., 2015, Quasi-static response of sandwich steel beams with corrugated cores, Engineering Structures, 97, 80-89 
19. Vinson J.R., 2001, Sandwich structures, Applied Mechanics Reviews, 54, 3, 201-214

20. Zhang J., QIN Q., WAng T.J., 2013, Compressive strengths and dynamic response of corrugated metal sandwich plates with unfilled and foam-filled sinusoidal plate cores, Acta Mechanica, 224, 4, 759-775

Manuscript received May 19, 2016; accepted for print September 8, 2016 